\title{
Simple polarimetric approach to direct measurement of the near-surface refractive index in graded-index films
}

\author{
Marcelo B. Pereira and Flavio Horowitz
}

\begin{abstract}
In the standard $M$-line method for the characterization of graded-index films, an analytical curve is fitted to the waveguide mode measurements and extrapolated to provide the refractive index in the zero-depth limit. Here we review our polarimetric approach to a direct near-surface measurement, which complements the $M$-line method. Also, we present its new and more straightforward version, which is applicable to existing samples and does not require masking before ion exchange. (C) 2003 Optical Society of America

OCIS codes: $310.6860,120.5410$.
\end{abstract}

Determination of the refractive index is essential in the design of optical systems and components and often requires nondestructive techniques. For graded-index waveguides manufactured by ion exchange, the index profile is obtained by the standard $M$-line technique, ${ }^{1}$ in which a continuous curve is fitted to experimental points that correspond to the guided light modes in the film, whereas the upper surface refractive index results from extrapolation of this curve to near-zero film depth. In fact, depth became the most critical region of disagreement when several laboratories, each using its own numerical treatment, applied the $M$-line measurement technique to the same ion-exchanged glass samples. ${ }^{2}$

In previous reports ${ }^{3-5}$ the present authors and others showed an alternative way to measure directly the near-surface refractive indices of graded-index waveguides. That way is a generalization of the Abelès method ${ }^{6}$ to inhomogeneous films, which uses the Hacskaylo extension ${ }^{7}$ for increased precision. This modified method is illustrated in Fig. 1(a). The absorbing layer permits matching of the reflectance from the coated region at the Brewster condition, i.e., at the substrate's back surface, to that of the sub-

The authors are with the Instituto de Física, Universidade Federal do Rio Grande do Sul, 91501-970 Porto Alegre, Rio Grande do Sul, Brazil. F. Horowitz's e-mail address is flavio@if.ufrgs.br.

Received 6 August 2002; revised manuscript received 13 February 2003.

0003-6935/03/163268-03\$15.00/0

(C) 2003 Optical Society of America strate's bare upper surface. Preparation for direct near-surface (DNS) measurement requires masking of a portion of the substrate (e.g., with an evaporated $\mathrm{Al}$ film) before the ion exchange, followed by mask removal before measurement.

To allow DNS measurement of existing coatings without previous masking, we developed a more straightforward method, as we describe here, based on the Brewster angle, ${ }^{8}$ with the Pfund procedure, ${ }^{9}$ for application to inhomogeneous films. As in the Abelès method, measurement of the Brewster angle uses $p$-polarized light to evaluate the refractive index, with typical precision to the second decimal place. Also, as in the Abelès-Hacskaylo method, addition of a slight amount of $s$-polarized light during the Brewster angle measurement (Pfund procedure) improves precision by 1 order of magnitude. Our scheme for measuring an arbitrary inhomogeneous film, provided that its interface with the incident medium (air) is the unique index discontinuity traversed by the reflected light, requires only that an absorbing layer be placed in the substrate's back surface, as shown in Fig. 1(b).

As discussed in Ref. 3, the Brewster condition attained in both DNS measurement schemes can be seen as resulting fundamentally from admittance matching at an interface and thus as having an interfacial nature. From now on we refer to our generalized Brewster-Pfund method as DNS-B.

The basic experimental setup is shown in Fig. 2, a detailed description of which can be found in Refs. $3-5$. In the DNS-B measurement, at each angle of incidence, $\varphi$, the azimuth angle of the analyzer trans- 

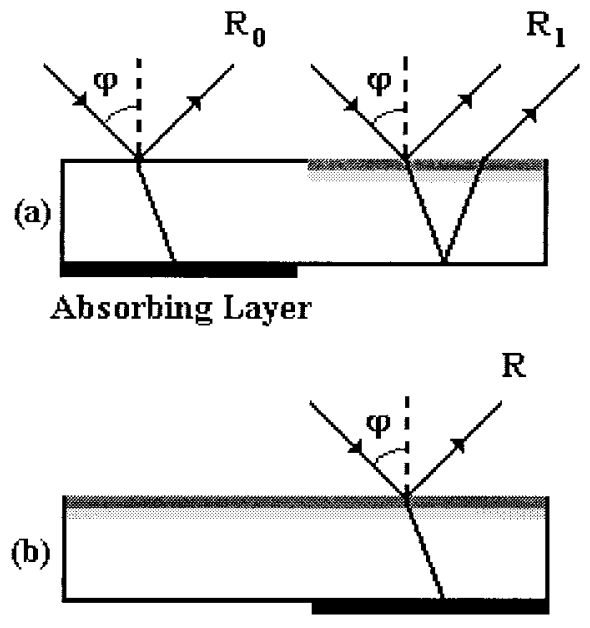

Absorbing Layer

Fig. 1. Illustration of our two DNS measurement schemes for inhomogeneous films: (a) generalized Abelès-Hacskaylo and (b) generalized Brewster-Pfund, both with an absorbing layer to extinguish undesired reflections. This extinction is verified when the weaker backreflectance beam, shifted from the front reflectance beam by propagation in the millimeter-thick substrate, disappears. The two methods provide practically the same results, indicating the consistency of the DNS approach.

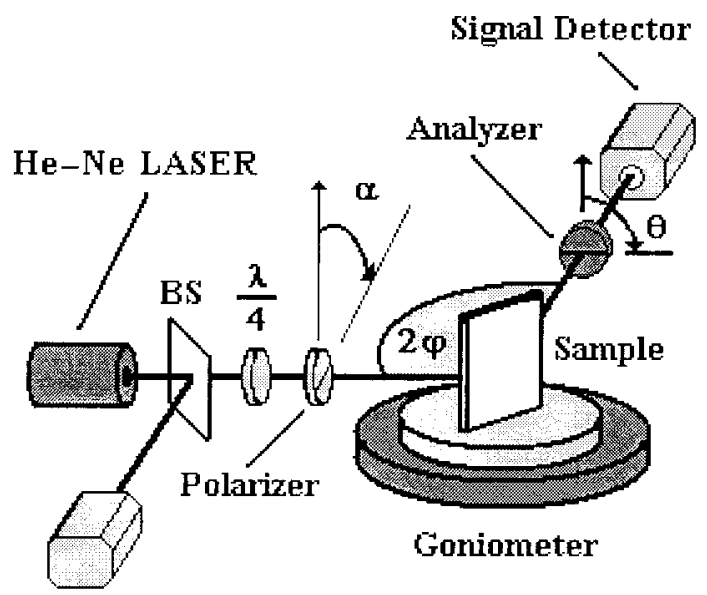

Ref. Detector

Fig. 2. Experimental setup. $\theta$ and $\varphi$, respectively the orientation of the analyzer and the angle of incidence, are directly obtained from the goniometer readings with a precision of 0.01 degree of arc. The other angle, $\alpha$, refers to the polarizer transmission axis, which deviates slightly from the plane of incidence. BS, beam splitter; Ref., reference.

mission axis, $\theta$, is set to reduce reflectance to almost zero.

Near the Brewster angle the relationship between $\varphi$ and $\theta$ becomes linear. At the Brewster angle the analyzer's transmission axis must be oriented parallel to the plane of incidence for light extinction, corresponding to $\theta=90^{\circ}$ in our convention. Figure 3 shows $\Omega=\left|\theta-90^{\circ}\right|$ versus $\varphi$, obtained by interpolation from a set of angular measurements performed

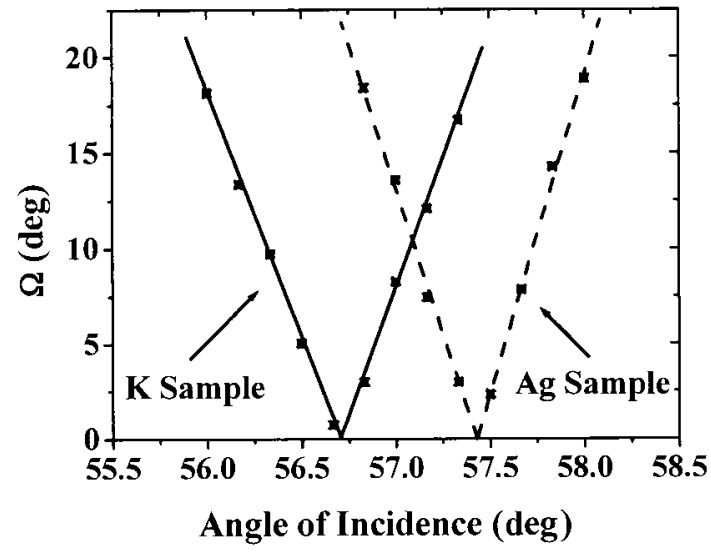

Fig. 3. Data and interpolation curves measured by the DNS-B method for Ag-exchanged and K-exchanged samples.

Table 1. Brewster Angles from Fig. 3, and Corresponding Near-Surface Film Refractive Indices, As Obtained by the DNS-B Method ${ }^{a}$

\begin{tabular}{lccc}
\hline Sample & $\begin{array}{c}\text { Brewster } \\
\text { Angle (deg) }\end{array}$ & $\begin{array}{c}\text { Refractive } \\
\text { Index }\end{array}$ & $\begin{array}{c}M \text {-Line } \\
\text { Refractive } \\
\text { Index }\end{array}$ \\
\hline $\mathrm{Ag}$ & $57.44 \pm 0.02$ & $1.566 \pm 0.001$ & $1.612 \pm 0.001$ \\
$\mathrm{~K}$ & $56.71 \pm 0.02$ & $1.523 \pm 0.001$ & $1.5393 \pm 0.0003$ \\
\hline
\end{tabular}

${ }^{a}$ Column 4 presents the zero-depth, extrapolated values produced by the $M$-line technique. ${ }^{10}$

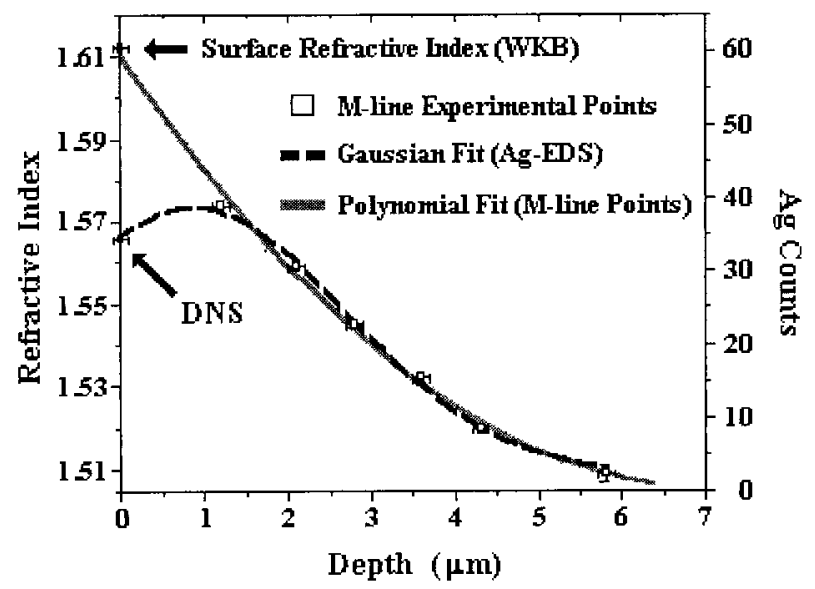

Fig. 4. Refractive-index values obtained by $M$-line (five waveguide modes) and by DNS-B (film and bare glass surfaces) measurement techniques. Energy-dispersion spectroscopy (EDS) values (five scans with 700 points each, $20-\mathrm{keV}$ electron acceleration voltage) are scaled to permit a Gaussian fit with small deviations from all $M$-line and DNS-B experimental values. This fit was set to overlap the index profile in the high depth region where the Ag concentration has its lowest values.

in $\mathrm{Ag}^{+}$and $\mathrm{K}^{+}$exchanged samples. Results are compiled in Table 1.

The discrepancy between DNS and extrapolated $M$-line results, as given in Table 1 , can be attributed to a depletion of the exchange salt concentration in 
the region close to the glass surface. ${ }^{11}$ Furthermore, we subjected the same samples to measurement by energy-dispersion spectroscopy, whose $\mathrm{Ag}$ counting profile is shown in Fig. 4, with the peak Ag concentration shifted from the surface by $\sim 0.9 \mu \mathrm{m}$.

This is further evidence of the interfacial character of DNS measurement, whereas the measured $M$-line value closest to the surface corresponds to a light mode with turning depth at $\sim 1.2 \mu \mathrm{m},{ }^{4}$ as well as of the complementary nature of the two methods. As in the original Brewster and Abelès methods (homogeneous films), our DNS methods for measurement of inhomogeneous films are valid for single-layer, nonabsorbing films.

In conclusion, a combination of DNS and $M$-line methods can bring us a more realistic picture of refractive-index profiles in ion-exchanged films. In particular, compared with the previous AbelèsHacskaylo method, the DNS-B approach demands less sample preparation and uses a simpler measurement procedure, making determination of the nearsurface refractive index significantly more practical and less time consuming.

We are grateful to G. C. Righini and S. Pelli of the Istituto di Ricerca sulle Onde Elettromagnetiche, Florence, for providing us the ion-exchange samples, and for support from the Brazilian agencies Coordenação de Aperfeiçoamento de Pessoal de Nível Superior and Programa de Apoio ao Desenvolvimento Científico e Tecnológico/Conselho Nacional de Desenvolvimento Científico e Tecnológico.

\section{References}

1. P. K. Tien, R. Ulrich, and R. J. Martin, "Modes of propagating light waves in thin deposited semiconductor films," Appl. Phys. Lett. 14, 291-294 (1969).

2. S. Pelli, G. C. Righini, A. Scaglione, G.-L. Yip, P. Noutsious, A.
Bräuer, P. Dannberg, J. Liñares, C. Gomez Reino, G. Mazzi, F. Gonella, R. Rimet, and I. Schanen, "Testing of Optical waveguides (TOW) cooperative project: preliminary results of the characterization of $k$-exchanged waveguides," in Linear and Nonlinear Integrated Optics, G. C. Righini and D. Yevick, eds., Proc. SPIE 2212, 126-131 (1994).

3. M. B. Pereira and F. Horowitz, "Optical surface analysis of graded index coatings on glass," J. Non-Cryst. Solids 218, 286 290 (1997).

4. F. Horowitz, M. B. Pereira, and M. Behar, "Analysis of $\mathrm{Ag}^{+}-$ exchanged glass coatings in the near-surface region," Opt. Commun. 182, 129-133 (2000).

5. F. Horowitz, M. B. Pereira, M. Behar, S. Pelli, and G. C. Righini, "Characterization of the near-surface region in ionexchange glass waveguides," in Optoelectronic Materials and Devices: Integrated Optics Devices V, G. C. Righini and S. Honkanen, eds., Proc. SPIE 4277, 99-104 (2001).

6. F. Abelès, "Recherches sur la propagation des ondes électromagnétiques sinusoidales dans les milieux stratifiés. Application aux couches minces," Ph.D. dissertation (Université de Paris Faculté des Sciences, Paris, 1949), pp. 1-120.

7. M. J. Hacskaylo, "Determination of the refractive index of thin dielectric films," J. Opt. Soc. Am. 54, 198-203 (1964).

8. S. Hacke, "Brewsterwinkel-Mikroskopie zur Untersuchung der Kristallisation von Calciumcarbonaten an ModellMonofilmen an der Grenzfläche Wasser/Luft," Ph.D. thesis (Georg-August-Universität zu Göttingen, Göttingen, Germany, 2001), pp. 1-59.

9. H. E. Bennett and J. M. Bennett, "Precision measurements in thin film optics," in Physics of Thin Films, Advances in Research and Development, G. Hass and R. E. Thun, eds. (Academic, New York, 1967), Vol. 4, pp. 1-90.

10. M. B. Pereira, "Optical metrology of surfaces and dielectric films, with extension to inhomogeneous coating microstructures," M.Sc. dissertation (Universidade Federal do Rio Grande do Sul, Porto Alegre, Brazil, 1998; in Portuguese), pp. 1-97.

11. T. G. Giallorenzi, E. J. West, R. Kirk, R. Ginhter, and R. A. Andrews, "Optical waveguides formed by thermal migration of ions in glass," Appl. Opt. 12, 1240-1245 (1973). 